Biodemography Soc Biol. 2018 ; 64(3-4): 173-186. doi:10.1080/19485565.2019.1579084.

\title{
Perceived Neighborhood Social Cohesion and Cardiometabolic Risk: A Gene x Environment Study
}

\author{
Jennifer W. Robinette ${ }^{a}$, Jason D. Boardman ${ }^{b}$, Eileen Crimmins ${ }^{a}$ \\ aDavis School of Gerontology, University of Southern California, 3715 McClintock Avenue, Los \\ Angeles, CA 90089-0191 \\ bInstitute of Behavioral Science and Department of Sociology University of Colorado, Boulder, \\ 1440 15th Street, Boulder, CO 80309
}

\begin{abstract}
People living in socially cohesive neighborhoods generally have better health. We extend this research by evaluating the hypothesis that perceived neighborhood cohesion may influence health by attenuating genetic liability for cardiometabolic risk factors. Using data from the Health and Retirement Study ( $\mathrm{n}=6615$; mean age 69.7), we conducted a gene $\mathrm{x}$ environment interaction study hypothesizing that perceived neighborhood cohesion would attenuate the link between polygenic scores for waist-to-hip ratio (WHR) and body mass index (BMI) and a measure of multi-system cardiometabolic risk (systolic and diastolic blood pressure, heart rate, A1c, Creactive protein, and total and HDL cholesterol). In support of the hypothesis, results indicated that among people perceiving low neighborhood cohesion, higher WHR polygenic scores were associated with greater cardiometabolic risk. In contrast, the genetic-cardiometabolic risk link was much attenuated among those living in neighborhoods perceived as socially cohesive. Our results support community-level interventions to enhance the social cohesiveness of individuals' neighborhoods which may provide health benefits by reducing the risks associated with known genetic risk factors.
\end{abstract}

\section{Keywords}

neighborhoods; genetics; cardiometabolic; interactions; aging

\begin{abstract}
People who live in neighborhoods where neighbors can trust and count on one another have lower risk for cardiovascular disease (CVD; Berkman and Kawachi 2000). Several physiological factors, such as elevated blood pressure, excessive physical weight, large waist circumference, and large waist-to-hip ratio (WHR) increase the risk of CVD, and according to the World Heart Federation, the likelihood of CVD increases with each additional physiological risk factor a person has (World Heart Federation, 2017). As such, there is growing interest in examining relationships between neighborhood features and summary
\end{abstract}

Send correspondence to Jennifer W. Robinette. jrobinet@ usc.edu; Davis School of Gerontology, University of Southern California, 3715 McClintock Avenue, Los Angeles, CA 90089-0191, U.S. 1-858-774-5254. jrobinet@usc.edu.

In accordance with Taylor \& Francis policy and my ethical obligation as a researcher, I am reporting that none of the authors have any conflicts of interests to report. 
measures of physiological risk that incorporate assessments of multiple physiological indicators (Robinette, Charles, Almeida, and Gruenewald 2016; Robinette, Charles, and Gruenewald 2017). Although individual indicators used in the construction of summary measures of physiological risk such as BMI and WHR are related, they uniquely contribute added risk to outcomes such as mortality (Pischon et al. 2008).

Moreover, these physiological risk factors are partially heritable (Allison et al. 1996; Doris 2011; Rose, Newman, Mayer-Davis, and Selby 1998). Specific genetic variants have been identified that are associated with elevated blood pressure and body mass index (BMI; Arora and Newton-Cheh 2010; Frayling et al. 2007). Wide ranging heritability estimates for these risk factors, however, suggest the possibility of gene-environment interactions $(\mathrm{GxE}$; Boardman et al. 2014) in which some individuals, despite increased genetic liability, avoid the progression to CVD. In the present study, we examined whether perceived social cohesion of people's immediate environment attenuates genetic risk for a constellation of these physiological risk factors (blood pressure, lipids, and inflammation) that, in turn, increase people's risk for the development of CVD. In particular, and given that BMI and WHR independently increase risk for death (Pischon et al. 2008), we interacted polygenic scores for both BMI and WHR with perceived neighborhood social cohesion. The genetic components comprising the BMI and WHR polygenic scores used in the present study are not only distinct from one another, but are also associated with multiple other metabolic traits (Locke et al. 2015; Shungin et al. 2015). These features make the BMI and WHR polygenic scores an ideal comparison in relation to a summary measure of cardiometabolic risk.

\section{Neighborhood Cohesion and Health}

Neighborhood cohesion has been related to higher self-rated health and helps to explain the link between higher levels of neighborhood socioeconomic status (SES) and better self-rated health (Franzini, Caughy, Spears, and Esquer 2005). Similarly, higher neighborhood cohesion has been associated with reduced physiological dysregulation and mediating links between higher neighborhood SES and lower physiological dysregulation (Schulz et al. 2013). People living in cohesive neighborhoods have fewer chronic health conditions (Berkman and Kawachi 2000) including a reduced risk of hypertension (Mujahid et al. 2008) and stroke (Kim, Park, and Petersen 2013).

Neighborhood cohesion has also been related to lower negative affect, higher positive affect, and fewer physical symptoms (Robinette, Charles, Mogle, and Almeida 2013). Moreover, people living in cohesive neighborhoods are less reactive to daily stressors, i.e., negative affect increased less in response to daily stressors. Given the wide range of health outcomes that have been correlated with neighborhood cohesion, coupled with evidence that cohesion buffers against stressors for people's well-being, we examined whether perceived neighborhood cohesion may further protect health by offsetting people's genetic risk for CVD-related outcomes.

This hypothesis is often called the "social control" gene-environment interaction model and it is described in great detail elsewhere (Boardman, Daw, Freese 2013). Environments may 
include people's behaviors, social relationships, or the larger contexts in which people are embedded. These environments may interact with people's genotypes in multiple ways (Shanahan and Hofer 2005), including when the environment may either buffer or exacerbate genetic predispositions for disease and disease-related factors.

Previous research has demonstrated that genetic liability for CVD risk factors is mitigated by individuals' health behaviors, for example by engaging in regular physical activity (for a review, see Lee, Lai, Ordovas, and Parnell 2011). However, individuals' behaviors are further contextualized by features of larger environments such as neighborhoods, for instance with the provision of outlets for physical activity or higher levels of cohesion, or lack thereof (Yi, Trinh-Shevrin, Yen, and Kwon 2016; Yip, Sarma, and Wilk 2016). The recent availability of genetic data in large national studies has allowed for investigations of GxE in a wider range of populations and environments, the latter of which can include the neighborhoods in which people live. We examined the hypothesis that greater perceived neighborhood social cohesion, perhaps through its stress-buffering (Robinette et al. 2013) and health behavior-promoting correlates (Yip et al. 2016), will attenuate genetic predisposition for physiological indicators of CVD.

\section{Biological CVD Risk}

CVD is the leading cause of death in the U. S. (Fuster 2010). Many biological factors increase the risk for CVD, including elevated blood pressure, BMI, WHR, lipids, and inflammation. Heritability estimates for these common CVD risk factors range from .05-.90 for BMI (e.g., Allison et al. 1996), .36-.61 for WHR (e.g., Rose et al. 1998), and .30-.70 for blood pressure (e.g., Doris 2011). These biological risk factors share many of the same genetic variants which may, in turn, partially explain their common co-occurrence (Andreasen et al. 2014).

Most complex traits including CVD-related outcomes are polygenic, or result from the influence of multiple genetic regions (Boyle, $\mathrm{Li}$, and Pritchard 2017). This has been shown in the case of cholesterol (Talmud et al. 2013), blood pressure (Andreasen et al. 2014), BMI (Abadi et al. 2017; Locke et al. 2015), and WHR (Shungin et al. 2015). As such, genetic epidemiologists have argued that more can be learned from summary genetic scores incorporating many markers than from candidate genes or any single nucleotide polymorphisms (SNP; Boardman et al. 2014). Summary genetic scores for measures of cardiometabolic risk, the key outcome in the present study, are currently unavailable. However, the present analyses used two polygenic scores, or summary measures representing the combined influence of multiple genetic variants on WHR and BMI to examine GxE interactions in relation to a measure of cardiometabolic risk. The BMI and WHR polygenic scores represent distinct genetic components, and are also associated with multiple other metabolic traits (Locke et al. 2015; Shungin et al. 2015).

WHR is a stronger predictor of CVD-related mortality than BMI, likely because this biological indicator captures body fat-mass and adipose tissue distribution (Leitzmann et al. 2011). BMI, which does not distinguish between fat- and non-fat mass, is elevated even among healthy athletes (Etchinson et al. 2011). WHR may therefore more accurately capture 
the consequence of poor health behaviors, such as unhealthful diet, inadequate physical activity, and alcohol consumption. Comparison of the polygenic scores for WHR and BMI in the present study allow for a better test of environmental influence, given that WHR is a better measure of the apple-shaped body thought to increase the risk of several cardiovascular diseases (Mayo Clinic 2018).

\section{GxE Interactions}

There is no paucity of evidence supporting GxE indicating that genetic predispositions for CVD risk factors are exacerbated by poor health behaviors (Lee et al. 2011). However, neighborhood cohesion is linked not only to better health (Berkman and Kawachi 2000) but also more health-enhancing behaviors (Yi et al. 2016). Focusing only on health behaviors as 'environment' thus limits understanding of the multi-level, social ecological contributors to health. In fact, interventions aimed at changing individuals' behavior may fail to improve health when the targeted behavior is shaped by conditions of the larger environment (Lonk and Phelan 1995).

Increasing attention has been paid to larger environments, including features of youths' schools that interact with genotypes to predict smoking (Boardman et al. 2008), alcohol consumption (Daw et al. 2013), and BMI (Boardman et al. 2012). Genotype-school interactions have indicated that heritability of smoking is higher among youth attending high schools in which popular students smoked (e.g., Boardman et al 2008). Further, high school peer tobacco and alcohol consumption was related to one's own consumption, but even more so among students more genetically sensitive to their environments (Daw et al. 2013). Taken together, school environments where smoking and alcohol consumption were normalized 'triggered' students' genetic risk for these behaviors.

Relatively less attention has been afforded to protective environmental resources, although some evidence suggests that school policies and resources aimed at maintaining a healthy weight attenuated the heritability of higher BMI (e.g., Boardman et al. 2012). Even less research has examined the possibility that neighborhood resources may interact with genetic risk for poor health, and those that have similarly focus on behavioral outcomes among youth (e.g., Beaver et al. 2011; Simons et al. 2011).

\section{The Present Study}

In the present analyses, we extend existing work on GxE for health in several ways. First, we define the 'environment' at the neighborhood level and focus on perceptions of social cohesion in the neighborhood as a key feature that shapes health and the ability to engage in a healthy lifestyle. Second, rather than focusing on neighborhood adversity as a potential 'trigger,' we examine the relatively unexplored question of whether perceived neighborhood cohesion can buffer against people's genetic risk for cardiometabolic risk. Third, we use a large sample of older adults to investigate a potential GxE in relation to a measure of multisystem cardiometabolic risk which heightens the development of CVD with age. Older adults represent a large and growing segment of the population, and CVD is disproportionately represented among older adults. Moreover, age-related declines in health 
and mobility may result in older adults' greater exposure and susceptibility to neighborhood features. Fourth, we use polygenic scores for WHR and BMI for a more stringent test of environmental influence on poor health. We include both scores to examine their relative contributions to cardiometabolic risk. We situate our analyses in the social control GxE typology described by others (Shanahan and Hofer 2005), where resources within people's environments may attenuate a genetic predisposition. We hypothesized that perceived neighborhood cohesion would attenuate genetic risk for WHR and BMI.

\section{Method}

\section{Participants and Procedures}

Data in the present study came from the Health and Retirement Study (HRS), a national survey examining the retirement and health status among men and women aged 50 years and older in the U. S. Economic, physical, mental, and cognitive well-being data have been collected every two years since 1992. Starting in 2006, a random half of households (excluding residents of nursing homes and other institutions) provided blood and saliva samples and completed physical exams. Results from the physical exam and blood samples were used to construct a measure of cardiometabolic risk. Buccal swabs collected in 2006 and saliva samples collected in 2008 were genotyped. These same participants completed psychosocial questionnaires including questions about their neighborhoods. The second half of sampled households completed these procedures in 2008. The first longitudinal biological and psychosocial follow-up was conducted in 2010 (from 2006) and 2012 (from 2008). All participants signed separate consent forms prior to providing biological samples and all research procedures were approved by the University of Michigan's Institutional Review Board.

The most recent biological and psychosocial data collected in 2010 and 2012 (combined for a complete sample) were included in the present analyses to examine GxE in relation to a measure of cardiometabolic risk. The analytic sample was restricted to those with useable genotyped data and who self-reported non-Hispanic white race/ethnicity $(n=9947)$; the polygenic scores used in the present analyses were constructed using data from nonHispanic whites and may therefore not have the same predictive ability among those from other racial/ethnic groups (Martin et al. 2017; Ware et 1. 2017a). Of those 9947 non-Hispanic white participants, 7800 have biological and exam data for the construction of a measure of cardiometabolic risk. Some participants did not respond to questions about neighborhood cohesion $(n=960)$ or household income $(n=225)$, resulting in an analytic sample of 6615 non-Hispanic whites.

\section{Measures}

\section{Biomarkers.}

Dried blood samples were assayed for total and high-density lipoprotein (HDL) cholesterol, C-reactive protein (CRP), and glycosylated hemoglobin (HbA1c). These biomarkers indicated lipid levels, glycemic control (and risk for insulin resistance) over the past two to three months, and general systemic inflammation, respectively (Crimmins et al. 2008). 
Results of the physical exam included assessments of systolic blood pressure (SBP), diastolic blood pressure (DBP), and pulse (HR). Each indicator variable was dichotomized into categories representing low risk (coded 0 ) and high risk (coded 1) using clinical cutpoints (see Table 1; Centers for Disease Control and Prevention 2016; Crimmins et al. 2014; National Heart, Lung, and Blood Institute 2015). A summary measure of cardiometabolic risk was constructed by summing across these seven dichotomous indicators with higher values demonstrating greater physiological risk (ranging from 0 indicating no indicators in the category of risk to seven where all indicators were in the category of risk).

\section{Perceived neighborhood cohesion.}

Participants rated social cohesion in their neighborhoods with four items (Mendes de Leon et al. 2009). Using a 7-point Likert-type scale, participants indicated their agreement with four statements: "I really feel a part of this area," "Most people in this area can be trusted," "Most people in this area are friendly," and "If you were in trouble, there are lots of people in this area who would help you." Reversed coded items were averaged so that higher scores represented greater neighborhood cohesion (Cronbach's $a=0.83$ ). As with previous literature (Aranda et al. 2011; Deutsch, Crockett, Wolff, and Russell 2012; Spencer, Cole, Jones, and Swanson 1997; Veling, Susser, Selten, and Hoek 2015), we used a dichotomous indicator of neighborhood cohesion to better differentiate between cohesive communities compared to all others.

\section{Genetic data.}

DNA was genotyped at the Center for Inherited Disease Research (CIDR) using the Illumina HumanOmni2.5-4v1 array, which measures roughly 2.4 million SNPs, of which roughly 1.9 million passed quality control procedures in the HRS (details regarding quality assurance/ quality control are found elsewhere; Weir 2012). Imputation to the 1000 Genomes Project, performed by the University of Washington Genetics Coordinating Center, yielded allele status on roughly 24 million SNPs (CIDR 2012). To adjust for potential population stratification, or that people from different racial/ethnic backgrounds may have differing genetic structures (Novembre and Stephens 2008), results from principal components analysis (PCA; Ware, Schmitz, Faul 2017) were included as covariates.

The WHR and BMI polygenic scores were constructed by the HRS staff in PLINK and PRSIce using all genotyped SNPs (no imputed SNPs and no clumping or pruning based on linkage disequilibrium; Ware et al. 2017b). Scores were constructed by summing the weighted beta estimates, which were determined from genome-wide association studies (GWAS) meta-analysis (Locke et al. 2015; Shungin et al. 2015). Reference alleles were flipped when beta estimates were negative so that higher scores were predictive of higher WHR or BMI. Final WHR and BMI polygenic scores were standardized ( mean $=0, \mathrm{sd}=1$ ).

\section{Additional covariates.}

A 2012 household income variable was used that summed across income from earnings, pensions and annuities, social security disability and retirement, unemployment and workers' compensation, government transfers, and household capital income for both the respondent and his or her spouse combined (see Bugliari et al. 2016 for a detailed 
description of the income variable and imputation processes). An income-to-needs variable was then constructed by dividing 2012 household income by U. S. family size-adjusted poverty threshold for 2012. This measure was used as a proxy for individual SES in the present analyses because of its inclusion of pension, social security, and retirement income, major sources of financial support among an older sample. We also considered years of schooling and wealth as alternative measures of individual SES. Wealth was calculated by summing across different assets (e.g., real estate, vehicles, businesses, individual retirement accounts, stocks, bonds, savings) less all sources of debt. Age and gender are included in analyses as covariates.

\section{Statistical analyses.}

Due to the complex survey design, we survey set the data and used the svy: suite of commands in Stata 13. We conducted a series of $t$ tests to compare sample characteristics of the analytic sample and the full HRS sample. To examine potential gene-environment correlation, with the presence of such a correlation introducing bias in hypothesized estimates of GxE, we also regressed the polygenic scores on perceptions of neighborhood cohesion and vice versa.

Given that cardiometabolic risk is calculated as a count variable in the present analyses, we used Poisson regressions to examine our hypotheses. These models were adjusted for income-to-needs, age, gender, and principal components. In our first model, we examined our hypothesis that higher polygenic scores for BMI and lower perceived neighborhood cohesion would each be related to greater cardiometabolic risk. In our second model we examined whether higher perceptions of neighborhood cohesion would attenuate the hypothesized genetic-cardiometabolic risk link (GxE). In an effort to properly adjust for individual-level confounds in our analysis of GxE (Keller, 2014), in Model 3 we further adjusted for six interactions between each of our primary covariates (age, gender, and income-to-needs) and our two primary predictors (perceptions of neighborhood cohesion and polygenic scores for BMI). Models 4-6 are identical to Models 1-3, but replace the polygenic score for BMI with the polygenic score for WHR. As a sensitivity analysis, models were also run with a continuous perceived neighborhood cohesion variable (and see supplemental Table 4 for results of these models run in an OLS regression).

\section{Results}

A description of the weighted sample can be found in Table 2. Participants generally had low levels of cardiometabolic risk, with only about 20 percent of the sample having three or more biological indicators with values above clinical cut-points. The neighborhoods represented in present sample were rated as having fairly high levels of cohesion.

We compared individuals in the analytic sample $(n=6615)$ and the remainder of the nonHispanic white individuals with useable genetic data $(n=3332)$ on the polygenic scores for BMI and WHR and observed no group differences $(t=-0.97, p=0.33 ; t=1.41, p=0.16$, respectively). The proportion of people perceiving low cohesion in their neighborhoods was larger in the analytic sample than the remainder of the sample $\left(x^{2}=1300, p<0.001\right)$. There was significantly lower cardiometabolic risk observed among those in the analytic sample ( $t$ 
$=5.81, p<0.001)$. Income-to-needs was similar in the two groups $(t=-0.74, p=0.46)$. The analytic sample was slightly younger $(t=5.67, p<.001)$, and the ratio of women to men was slightly larger in the analytic sample $\left(\mathrm{x}^{2}=7.44, p<0.01\right)$. Results of our regression analyses examining potential gene-environment correlation indicated that WHR polygenic scores neither predicted, nor was predicted by perceptions of neighborhood cohesion $(p=$ 0.11 and $p=0.11$, respectively).

Results from a series of regression models designed to evaluate our primary hypothesis are presented in Table 3. We begin by examining relationships between perceived neighborhood cohesion and the polygenic score for BMI and cardiometabolic risk in Model 1. Results indicated that higher BMI polygenic scores $(b=0.07, p<0.001)$ significantly related to greater cardiometabolic risk. Men and older adults had significantly higher cardiometabolic risk. Although perceived neighborhood cohesion was not a significant predictor of cardiometabolic risk in the dichotomous form, in the continuous form greater perceptions of neighborhood cohesion were significantly associated with lower cardiometabolic risk ( $b=$ $-0.03, p=0.02$ ). In Model 2 we added the perceived neighborhood cohesion x BMI polygenic score interaction. This interaction was not significant in either Model 2 or Model 3 with either a dichotomous or continuous perceived neighborhood cohesion variable.

In Model 4 we examined our hypotheses regarding the polygenic scores for WHR. Results indicated that people with higher polygenic scores for WHR had significantly greater cardiometabolic risk $(b=0.04, p=0.01)$. Results of Model 5 indicated a significant interaction such that, among people living in neighborhoods perceived as having low levels of cohesion, higher WHR polygenic scores were associated with greater cardiometabolic risk. Among those living in neighborhoods perceived as more cohesive, however, the WHR polygenic score-cardiometabolic risk relationship was attenuated $(b=-0.07, p=0.04$; see Figure 1). This pattern also emerged in a model using a continuous perceived neighborhood cohesion variable $(b=-0.03, p=0.04)$. The interaction persisted, but was somewhat attenuated when covariate $\mathrm{x}$ perceived cohesion and covariate $\mathrm{x}$ polygenic score interactions were introduced in Model $6(\mathrm{~b}=-0.06, p=0.05)$.

\section{Discussion}

In the present study, we constructed a summary measure of CVD-related biomarkers to characterize physiological risk among a nationally representative sample of non-Hispanic white older men and women in the U. S. Some people carry genetic characteristics that increase their risk for these individual biological CVD risk factors (Arora and Newton-Cheh 2010; Frayling et al. 2007) and therefore CVD outcomes. Considering the importance of the larger context in which people live, however, we examined a potential neighborhood-level GxE, hypothesizing that perceived neighborhood social cohesion, serving as an environmental support, may attenuate people's genetic liability. Although perceptions of neighborhood cohesion were not significantly associated with cardiometabolic risk, we observed a significant interaction; perceived neighborhood cohesion may offset genetic proclivity for a common set of biological CVD risk factors. This is the first investigation, to our knowledge, of a neighborhood-genotype interaction in relation to cardiometabolic risk, an important risk factor for one of the leading causes of death worldwide. 


\section{Perceived Neighborhood Cohesion}

We did not observe a significant main effect of perceived neighborhood cohesion on cardiometabolic risk. Others have similarly obtained null results in examinations of neighborhood cohesion and comparable measures of physiological risk (Robinette, Charles, Almeida, and Gruenewald 2016). People with higher WHR polygenic scores had greater cardiometabolic risk. We also observed a significant interaction between perceived neighborhood cohesion and WHR polygenic scores in relation to cardiometabolic risk. Higher polygenic scores that predispose people to higher WHR (and other correlated biological CVD risk factors) were more strongly linked to cardiometabolic risk among those perceiving their neighborhoods as not cohesive. Among those living in neighborhoods perceived as cohesive, WHR polygenic scores were not as predictive of cardiometabolic risk. These results support sentiments forwarded by others (Bookman et al. 2012) that main effects may not be observed (i.e., perceived neighborhood cohesion in the present study) when significant interactions, including significant cross-over effects, are operating.

\section{BMI and WHR Polygenic Scores}

$\mathrm{BMI}$ is a widely assessed measure of metabolic functioning hypothesized to increase the risk of CVD. This measure is limited, however, by its reliance on the ratio of height to weight, which does not take into account percentage or distribution of body fat. As such, a greater number of healthy athletes are categorized as obese using this measure when compared to WHR (Leitzmann et al. 2011). One of the primary aims of the present study was to compare BMI and WHR polygenic scores in their potential interaction with perceived neighborhood cohesion in relation to cardiometabolic risk.

Results of the present study indicated that both WHR and BMI polygenic scores significantly predicted cardiometabolic risk. Although the interaction between the WHR polygenic score and perceived neighborhood cohesion was observed, there was no significant interaction between BMI polygenic scores and perceived neighborhood cohesion in relation to cardiometabolic risk. This null result may be indicative of the aforementioned challenges inherent in the construction of BMI, namely that an excess of people is categorized as overweight or obese when considering only the ratio of height to weight. We predicted that perceived neighborhood cohesion would be protective against genetic risk for common indicators of CVD. BMI may be an inferior measure of lifestyle-related metabolic dysfunction relative to WHR, however, making sense of this null interaction.

\section{Limitations and Future Directions}

Results of the present study provide preliminary evidence for neighborhood-level attenuation of people's genetic proclivity for biological CVD risk factors. Moreover, these results indicate the relative contribution of WHR over BMI to physical health and provide further support for lifestyle choices and their relation to physical health. Given the paucity of work involving investigations of neighborhood-genotype interactions, however, replication is needed, and future work should include a wider range of neighborhood-level resources and health outcomes. Relatedly, neighborhood cohesion was self-reported in the present study. As a secondary analysis, we compared our results to those from a model that used another household member's ratings of neighborhood cohesion. The GxE was not significantly in 
this alternative model (results not shown). It is important to note that over 2000 non-married participants were lost in this alternative model, reducing statistical power to detect small neighborhood and genetic effects. Comparison of the cohesion ratings of individuals from the same household were significantly different in this sample $\left(x^{2}{ }_{(1)}=422.10, p<.0001\right)$. It is possible that perceptions of one's neighborhood are so unique to the individual that husbands and wives, for example, may view their neighborhoods in different ways and furthermore differentially benefit from perceived cohesion depending on their unique levels of neighborhood embeddedness. These individualized perceptions are likely relevant to people's health above and beyond the perceptions of other household members. Future replication of this work should nevertheless incorporate non-household member ratings of neighborhood cohesion, and perhaps measures that quantify networks of social interactions among neighbors. Increased availability of genetic data in national samples has allowed for the conduct of individual difference models, including the present analyses, that have not previously been possible. That said, generalizability is limited when samples are restricted so single racial/ethnic groups. An important goal for future research, as the field continues to understand how population stratification affects genetic structure and function, will be increased inclusiveness in $\mathrm{GxE}$ research that utilizes polygenic scores.

\section{Conclusions}

Findings from this study support the social control model of GxE (Shanahan and Hofer 2005), and suggest that perceived neighborhood cohesion potentially protects people against their own disease-relevant genetic characteristics. The policy implication inherent in this work is that cultivating cohesion among neighborhoods may assist in slowing communitylevel CVD development, also representing an important goal in public health. Although people's genotypes are not likely to be modified in this lifetime, policy change and community-level interventions have the potential to improve the social features of people's neighborhoods and therefore health (Bookman et al. 2012).

\section{Supplementary Material}

Refer to Web version on PubMed Central for supplementary material.

\section{Acknowledgments}

This work was supported by the National Institutes of Health/National Institute on Aging under grants T32AG000037-37, 1K99AG055699-01, and R25 AG053227.

\section{References}

Abadi A, Alyass A, Robiou du Pont S, Bolker B, Singh P,Mohan V,...Meyre D 2017 "Penetrance of polygenic obesity susceptibility loci across the body mass index distribution." American Journal of Genetics 101, 925-938. doi: 10.1016/j.ajhg.2017.10.007

Allison DB, Kaprio J, Korkeila M, Koskenvuo M, Neale MC, and Hayakawa K 1996 "The heritability of body mass index among an international sample of monozygotic twins reared apart." International Journal of Obesity and Related Metabolic Disorders 20, 501-506. [PubMed: 8782724]

Andreasen OA, McEvoy LK, Thompson WK, Wang Y, Reppe S, Schork AJ,... International Consortium for Blood Pressure Genome-Wide Association Studies. 2014 "Identifying common 
genetic variants in blood pressure due to polygenic pleiotropy with associated phenotypes." Hypertension 63, 819-826. doi: 10.1161/HYPERTENSIONAHA.113.02077 [PubMed: 24396023]

Aranda MP, Ray LA, Snih S, Ottenbacher KJ, and Markides KS 2011 "The protective effect of neighborhood composition on increasing frailty among older Mexican Americans: a barrio advantage?" Journal of aging and health 23, 1189-1217. [PubMed: 21948774]

Arora P and Newton-Cheh C 2010 "Blood pressure and human genetic variation in the general population." Current Opinion in Cardiology 25, 229-237. doi: 10.1097/HCO.0b013e3283383e2c [PubMed: 20224392]

Beaver KM, Gibson CL, DeLisi M, Vaughn MG, and Wright JP 2011 "The interaction between neighborhood disadvantage and genetic factors in the prediction of antisocial outcomes." Youth Violence and Juvenile Justice 10, 25-40. 10.1177/1541204011422085

Berkman LF and Kawachi I 2000 Social epidemiology New York, New York: Oxford University Press.

Boardman JD, Daw J, and Freese J 2013 "Defining the environment in gene-environment research: Lessons from social epidemiology.” American Journal of Public Health 103, S64-S72. doi: 10.2105/AJPH.2013.301355 [PubMed: 23927514]

Boardman JD, Domingue BW, Blalock CL, Haberstick BC, Harris KM, and McQueen MB 2014 "Is the gene-environment interaction paradigm relevant to genome-wide studies? The case of education and body mass index.” Demography 51, 119-139. doi: 10.1007/s13524-013-0259-4 [PubMed: 24281739]

Boardman JD, Saint Onge JM, Haberstick BC, Timberlake DS, and Hewitt JK 2008 "Do schools moderate the genetic determinants of smoking?" Behavioral Genetics 38, 234-246. doi: 10.1007/ s10519-008-9197-0

Boardman JD, Roettger ME, Domingue BW, McQueen MB, Haberstick BC, and Harris KM 2012 "Gene-environment interactions related to body mass: School policies and social context as environmental moderators." Journal of Theoretical Politics 24, 370-88. [PubMed: 23236222]

Bookman EB, McAllister K, Gillanders E, Wanke K, Balshaw D, Rutter J... Birnbaum LS 2012 "Gene-environment interplay in common complex diseases: Forging an integrative modelrecommendations from an NIH workshop.” Genetic Epidemiology 35, 217-225. doi: 10.1002/ gepi.20571

Boyle EA, Li YI, and Pritchard JK 2017 "An expanded view of complex traits: From polygenic to omnigenic.” Cell 169, 1177-1186. DOI: 10.1016/j.cell.2017.05.038 [PubMed: 28622505]

Bugliari D, Campbell N, Chan C, Hayden O, Hurd M, Main R...St. Clair P 2016 RAND HRS Data Documentation (Version P) http://hrsonline.isr.umich.edu/modules/meta/rand/randhrsp/ randhrs_P.pdf (accessed August 1, 2018).

Centers for Disease Control and Prevention, Division of Nutrition, Physical Activity, and Obesity. 2016 Defining adult overweight and obesity https://www.cdc.gov/obesity/adult/defining.html (accessed August 1, 2018).

Center for Inherited Disease Research, Health and Retirement Study. 2012 Imputation report - 1000 Genomes project reference panel http://hrsonline.isr.umich.edu/sitedocs/genetics/ HRS_1000G_IMPUTE2_REPORT_AUG2012.pdf (accessed August 1, 2018).

Crimmins E, Guyer H, Langa K, Ofstedal MB, Wallace R and Weir D 2008 "Documentation of physical measures, anthropometrics, and blood pressure in the Health and Retirement Study." https://hrs.isr.umich.edu/publications/biblio/5734 (accessed August 1, 2018).

Crimmins E, Kim JK, McCreath H, Faul J, Weir D, and Seeman T 2014 "Validation of blood-based assays using dried blood spots for use in large population studies." Biodemography and Social Biography 60, 38-48. doi: 10.1080/19485565.2014.901885

Daw J, Boardman JD, Peterson R, Smolen A, Haberstick BC Ehringer MA, Ennett ST, and Foshee VA 2014 "The interactive effect of neighborhood peer cigarette use and 5HTTLPR genotype on individual cigarette use.” Addictive Behaviors 39,1804-10. [PubMed: 25127196]

Deutsch AR, Crockett LJ, Wolff JM, and Russell ST 2012 "Parent and peer pathways to adolescent delinquency: Variations by ethnicity and neighborhood context." Journal of youth and adolescence 41, 1078-1094. [PubMed: 22460729] 
Doris PA 2011 "The genetics of blood pressure and hypertension: The role of rare variation." Cardiovascular Therapeutics 29, 37-45. doi:10.1111/j.1755-5922.2010.00246.x [PubMed: 21129164]

Etchinson WC, Bloodgood EA, Minton CP, Thompson NJ, Collins MA, Hunter SC, and Dai H 2011 "Body mass index and percentage of body fat as indicators for obesity in an adolescent athletic population." Sports Health 3, 249-252. doi: 10.1177/1941738111404655 [PubMed: 23016014]

Franzini L, Caughy M, Spears W, and Esquer MEF 2005 "Neighborhood economic conditions, social processes, and self-rated health in low-income neighborhoods in Texas: A multilevel latent variables model." Social Science and Medicine 61, 1135-1140. 10.1016/j.socscimed.2005.02.010 [PubMed: 15970226]

Frayling TM, Timpson NJ, Weedon MN, Zeggini E, Freathy RM,Lindgren CM, ...McCarthy MI 2007 "A common variant in the FTO gen eis associated with body mass index and predisposes to childhood and adult obesity." Science 316, 889-894. [PubMed: 17434869]

Fuster V 2010 "Epidemiology of cardiovascular disease." In Promoting cardiovascular health in the developing world: A critical challenge to achieve global health, ed. Fuster V and Kelly BB, Washington, DC: National Academies Press.

Keller M (2014). Gene x environment interaction studies have not properly controlled for potential confounders: The problem and the (simple) solution. Biological Psychiatry, 75, 18-24. 10.1016/ j.biopsych.2013.09.006 [PubMed: 24135711]

Kim ES, Park N, and Petersen C 2013 "Perceived neighborhood social cohesion and stroke." Social Science and Medicine 97, 49-55. 10.1016/j.socscimed.2013.08.001 [PubMed: 24161088]

Lee YC, Lai CQ, Ordovas JM, and Parnell LD 2011 “A database of gene-environment interactions pertaining to Blood lipid traits, cardiovascular disease and type 2 diabetes.” Journal of Data Mining and Genomics Proteomics 2.

Leitzmann MF, Moore SC, Koster A, Harris TB, Park Y, Hollenbeck A, et al. 2011 "Waist circumference as compared with body-mass index in predicting mortality from specific causes." PLoS ONE 6, e18582 10.1371/journal.pone.0018582 [PubMed: 21541313]

Locke AE, Kahali B, Berndt SI, Justice AE, Pers TH, Day FR, ... and Croteau-chonka DC 2015 "Genetic studies of body mass index yield new insights for obesity biology." Nature 518, 197-206. [PubMed: 25673413]

Lonk BG and Phelan J 1995 "Social conditions as fundamental causes of disease." Journal of Health and Social Behavior 80-95. [PubMed: 7560851]

Mayo Clinic. 2018 “Apple and pear body shapes.” https://www.mayoclinic.org/diseases-conditions/ metabolic-syndrome/multimedia/apple-and-pear-body-shapes/img-20006114 (accessed August 1, 2018).

Martin AR, Gignoux CR, Walters RK, Wojcik GL, Neale BM, Gravel S, ... and Kenny EE 2017 "Human demographic history impacts genetic risk prediction across diverse populations." The American Journal of Human Genetics 100(4), 635-649. [PubMed: 28366442]

Mendes de Leon CF, Cagney KA, Bienias JL, Barnes LL, Skarupski KA, Scherr PA, and Evans DA 2009 "Neighborhood social cohesion and disorder in relation to walking in community-dwelling older adults: A multi-level analysis." Journal of Aging and Health 21, 155-171. doi: 10.1177/0898264308328650 [PubMed: 19144973]

Mujahid MS, Diez Roux AV, Morenoff JD, Raghunathan TE, Cooper RS, Ni H, and Shea S 2008 "Neighborhood characteristics and hypertension." Epidemiology 19, 590-8. [PubMed: 18480733]

Novembre J and Stephens M 2008 "Interpreting principal component analyses of spatial population genetic variation.” Nature Genetics 40, 646-649. doi: 10.1038/ng.139 [PubMed: 18425127]

Pischon T, Boeing H, Hoffmann K, Bergmann M, Schulze MB, Overvad K... Riboli E 2008 "General and abdominal adiposity and risk of death in Europe.” New England Journal of Medicine 13, 2105-2120. doi: 10.1056/NEJMoa0801891

Robinette JW, Charles ST, Almeida DA, and Gruenewald TL 2016 "Neighborhood features and physiological risk: An examination of allostatic load." Health and Place 41, 110-118. doi: 10.1016/j.healthplace.2016.08.003 [PubMed: 27583527] 
Robinette JW, Charles ST, and Gruenewald TL 2017 "Neighborhood cohesion, neighborhood disorder, and cardiometabolic risk." Social Science and Medicine 198, 70-76. doi: 10.1016/j.socscimed. 2017.12.025 [PubMed: 29276988]

Robinette JW, Charles ST, Mogle JA, and Almeida D 2013 "Neighborhood cohesion and daily wellbeing: Results from a diary study." Social Science and Medicine 96, 174-182. doi: 10.1016/ j.socscimed.2013.07.027 [PubMed: 24034965]

Rose KM, Newman B, Mayer-Davis EJ, and Selby JV 1998 "Genetic and behavioral determinants of waist-hip ratio and waist circumference in women twins." Obesity Research 6, 383-392. [PubMed: 9845227]

Schulz AJ, Mentz G, Lachance L, Zenk SN, Johnson J, Stokes C, and Mandell R 2013 "Do observed or perceived characteristics of the neighborhood environment mediate associations between neighborhood poverty and cumulative biological risk?" Health and Place 24,147-56. [PubMed: 24100238]

Shanahan MJ and Hofer SM 2005 "Social context in gene-environment interactions: retrospect and prospect.” Journals of Gerontology: Psychological Sciences and Social Sciences 60, 65-76. [PubMed: 15863711]

Shungin D, Winkler TW, Croteau-Chonka DC, Ferreira T, Locke AE, Mägi R, ... and Workalemahu T 2015 "New genetic loci link adipose and insulin biology to body fat distribution." Nature 518, 187. [PubMed: 25673412]

Simons RL, Lei MK, Beach SR, Brody GH, Philibert RA, and Gibbons FX 2011 "Social environmental variation, plasticity genes, and aggression: evidence for the differential susceptibility hypothesis.” American Sociological Review 76, 833-912. [PubMed: 22199399]

Spencer MB, Cole SP, Jones SM, and Swanson DP 1997 "Neighborhood and family influences on young urban adolescents' behavior problems: A multisample, multisite analysis.” Neighborhood poverty $1,200-218$.

Talmud PJ, Shah S, Whittall R, Futema M, Howard P, Cooper JA,... Humphries SE 2013 "Use of lowdensity lipoprotein cholesterol gene score to distinguish patients with polygenic and monogenic familiar hypercholesterolaemia: A case-control study." The Lancet 381, 13-19. 10.1016/ S0140-6736(12)62127-8

U. S. Department of Health and Human Services, National Institutes of Health, National Heart, Lung, and Blood Institute. 2015 "Diagnosis of high blood pressure." https://www.nhlbi.nih.gov/health/ health-topics/topics/hbp/diagnosis (accessed on August 1, 2018).

Veling W, Susser E, Selten J-P, and Hoek HW 2015 "Social disorganization of neighborhoods and incidence of psychotic disorders: a 7-year first-contact incidence study.” Psychological medicine 45, 1789-1798. [PubMed: 25402968]

Ware E, Schmitz L, and Faul J 2017b "HRS Polygenic Scores: 2006-2010 Genetic Data.” https:// hrs.isr.umich.edu/news/hrs-polygenic-scores-2006-2010-genetic-data (accessed on August 1, 2018).

Ware EB, Schmitz LL, Faul JD, Gard AM, Mitchell CM, Smith JA, Zhao W, Weir DR, Kardia SLR. 2017a "Heterogeneity in polygenic scores for common human traits." BiorXiv doi: https:// www.biorxiv.org/content/early/2017/02/05/106062 (accessed August 1, 2018).

Weir DR 2012 “Quality Control Report for Genotypic Data.” http://hrsonline.isr.umich.edu/sitedocs/ genetics/HRS_QC_REPORT_MAR2012.pdf (accessed August 1, 2018).

World Heart Federation. 2017 “Risk factors." https://www.world-heart-federation.org/resources/riskfactors/ (accessed August 1, 2018).

Yi S, Trinh-Shevrin C, Yen IH, and Kwon SC 2016 "Neighborhood social cohesion and meeting physical activity guidelines: Does the association differ by race/ethnicity?" Circulation 133, A11.

Yip C, Sarma S, Wilk P 2016 "The association between social cohesion and physical activity in Canada: A multilevel analysis." Social Science and Medicine: Population Health 2, 718-723. 


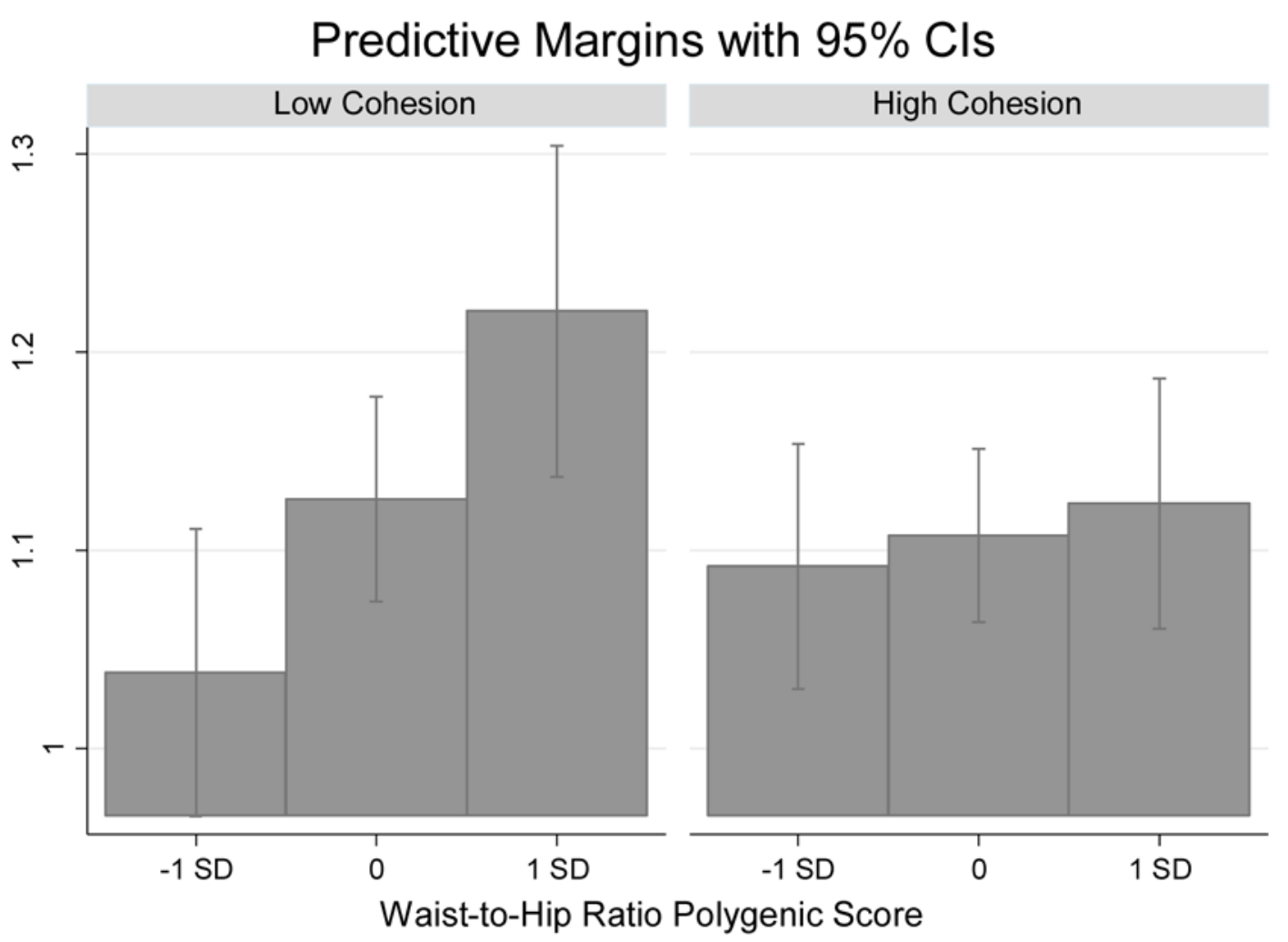

Figure 1.

Neighborhood cohesion $\mathrm{x}$ WHR polygenic score interaction 


\section{Table 1}

Clinical cut-points

\begin{tabular}{lc}
\hline Total Cholesterol & $240 \mathrm{mg} / \mathrm{dL}$ \\
HDL Cholesterol & $40 \mathrm{mg} / \mathrm{d}$ \\
Glycated Hemoglobin (HbA1c) & $6.5 \%$ \\
C-Reactive Protein & $3.0 \mathrm{mg} / 1$ \\
Systolic Blood Pressure & 140 \\
Diastolic Blood Pressure & 90 \\
Heart Rate & 90 \\
\hline
\end{tabular}




\section{Table 2}

Weighted sample characteristics

\begin{tabular}{lccc}
\hline & Overall & Low Cohesion & High Cohesion \\
& $\boldsymbol{M}(\mathrm{SE})$ & $\boldsymbol{M}(\mathbf{S E})$ & $\boldsymbol{M}$ (SE) \\
\cline { 2 - 4 } & $1.14(0.02)$ & $1.16(0.03)$ & $1.13(0.02)$ \\
Cardiometabolic Risk & 34.13 & 33.86 & 34.32 \\
No Biomarkers at Risk, \% & 32.86 & 32.66 & 33.00 \\
One Biomarker at Risk, \% & 21.14 & 21.18 & 21.10 \\
$\quad$ Two Biomarkers at Risk, \% & 11.87 & 12.30 & 11.58 \\
Three or More Biomarkers at Risk, \% & $0.00(0.01)$ & $0.03(0.02)$ & $-0.01(0.02)$ \\
HR Polygenic Score & $-0.01(0.01)$ & $0.04(0.02)$ & $-0.03(0.02)$ \\
BMI Polygenic Score & $5.62(0.02)$ & & \\
Neighborhood Cohesion & $4.75(0.13)$ & $4.54(0.19)$ & $4.90(0.17)$ \\
Household Income-to-Needs & $69.71(0.12)$ & $68.48(0.18)$ & $70.56(0.17)$ \\
Age, years & 46.44 & 49.35 & 44.87 \\
Gender, \% male & & & \\
\hline
\end{tabular}



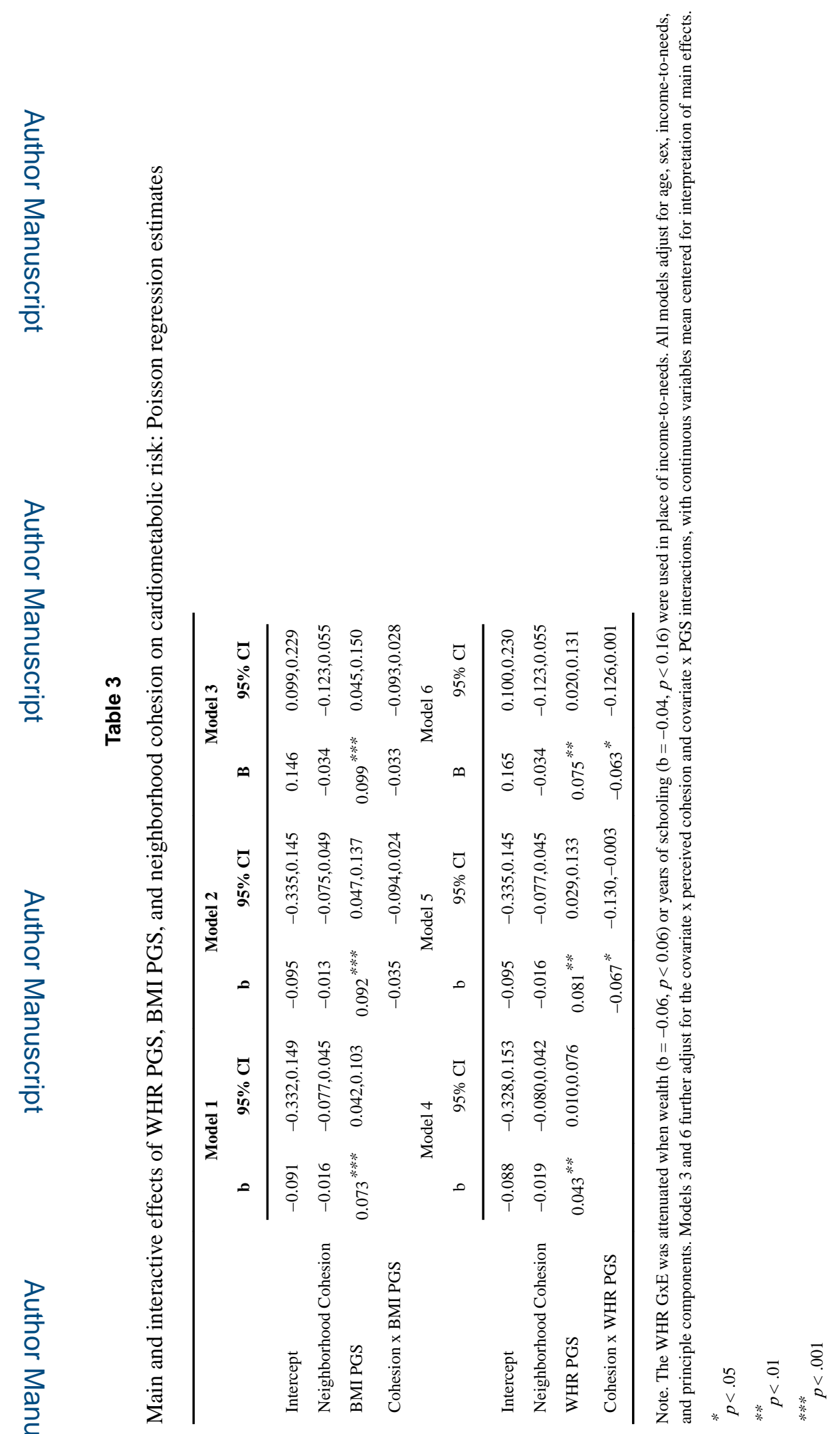

Biodemography Soc Biol. Author manuscript; available in PMC 2019 December 23. 